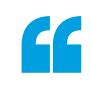

\section{a true 2D \\ magnetic \\ material is \\ interesting \\ because it \\ provides a \\ platform \\ on which}

questions can

be answered

\title{
Peeling off magnetic layers
}

The chemical functionalization of inorganic $2 \mathrm{D}$ materials in a homogeneous and defect-free manner is synthetically challenging and as a result applications that require high-quality materials are often not possible. This modification step generally occurs after the preparation of such materials, and is hampered by their low solubility and poor reactivity.

Now, writing in Nature Chemistry, Guillermo Mínguez, Eugenio Coronado and colleagues report the fabrication of defect-free 2D materials by the mechanical exfoliation of coordination polymers. Using a pre-synthetic approach, a series of coordination polymers with various functional groups are sufficiently robust to be mechanically exfoliated to produce five chemically distinct 2D materials. These metal-organic magnetic materials retain their long-range crystalline order after mechanical exfoliation and show high chemical stability in ambient conditions. These attributes are in contrast to those of existing 2D magnetic materials of an inorganic nature.

The layered coordination polymers are composed of $\mathrm{Fe}$ (II) centres and benzimidazole ligands with either $\mathrm{Cl}, \mathrm{H}, \mathrm{CH}_{3}, \mathrm{Br}$ or $\mathrm{NH}_{2}$ side groups. Using plastic tape, the functionalized bulk crystals are exfoliated and flakes of the 2D material deposited on a silicon substrate. The flakes are large in their lateral size $(>1 \mu \mathrm{m})$ and their thicknesses range from a single layer to hundreds of nanometres. In addition, this pre-synthetic approach and the mechanical, rather than liquid-based, exfoliation method produces functional 2D materials free from solvent and physisorbed molecules.

The researchers showed that the 2D materials made from the five different coordination polymers have differing surface wettability - ranging from hydrophobic for the halide side groups to super-hydrophilic for the $\mathrm{NH}_{2}$ side

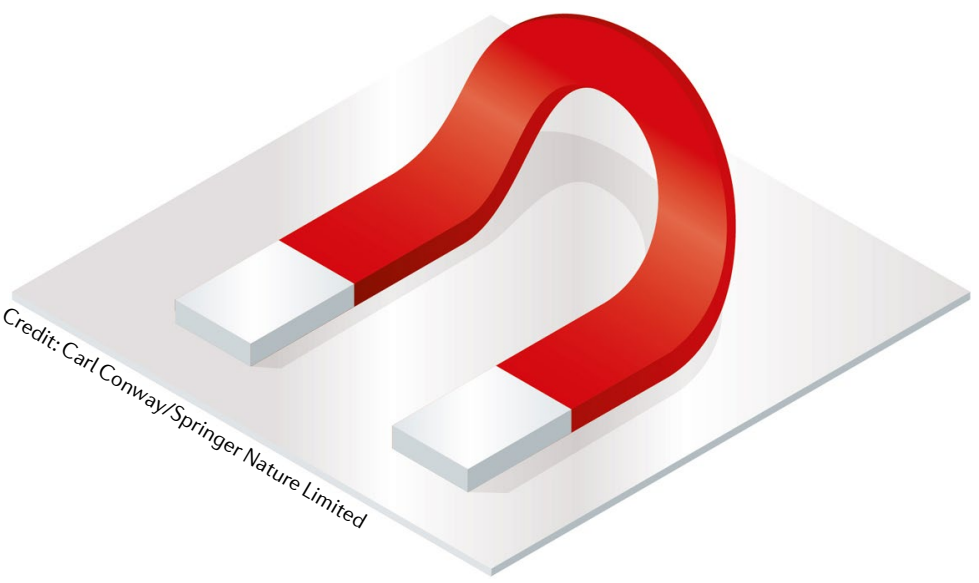

group. "The change of wettability that we show may be crucial for the fabrication of complex stacked heterostructures," added Mínguez.

As a consequence of the close proximity of neighbouring $\mathrm{Fe}(\mathrm{II})$ atoms, the layered materials - even when approaching the 2D limit show antiferromagnetic ordering with spin canting. "The magnetic properties of a material depend strongly on its dimensionality," explains Coronado. "Thus, the existence of a true 2D magnetic material is interesting because it provides a platform on which questions can be answered, such as the existence, or not, of long-range magnetic ordering in exchangecoupled 2D systems."

As well as allowing exploration of magnetism in the $2 \mathrm{D}$ limit, these 2D magnetic materials may enable more practical uses as mechanical membranes. "The versatile functionalization combined with the extraordinary mechanical properties of these 2D coordination polymers opens up the possibility to prepare ultrathin membranes that can recognize specific molecules and act as selective membranes for gas sensing," adds Mínguez.

"Finally, these ultrathin layers may be of interest in spin-electronic (spintronic) devices as they may act as magnetic tunnelling barriers or spin filtering layers in molecular spin valves," says Coronado.

Alison Stoddart

ORIGINAL ARTICLE López-Cabrelles, J. et al. Isoreticular two-dimensional magnetic coordination polymers prepared through presynthetic ligand functionalization. Nat. Chem. 10,1001-1007 (2018) 